\title{
Generation and Characterization of a Neutralizing Human Monoclonal Antibody to Hepatitis B Virus PreS1 from a Phage-Displayed Human Synthetic Fab Library
}

\author{
Gyunghee Jo ${ }^{1 \dagger}$, Mun Sik Jeong ${ }^{1 \dagger}$, Jimin $\mathrm{Wi}^{2}$, Doo Hyun Kim ${ }^{3}$, Sangkyu Kim ${ }^{1}$, Dain Kim ${ }^{1}$, Jun-Yeol Yoon ${ }^{1}$, \\ Heesu Chae ${ }^{1}$, Kyun-Hwan Kim ${ }^{3,4}$, and Hyo Jeong Hong ${ }^{1,2 *}$ \\ ${ }^{1}$ Department of Systems Immunology, College of Biomedical Science, Kangwon National University, Chuncheon 24341, Republic of Korea \\ ${ }^{2}$ Scripps Korea Antibody Institute, Chuncheon 24341, Republic of Korea \\ ${ }^{3}$ Department of Pharmacology, Center for Cancer Research and Diagnostic Medicine, IBST, School of Medicine, Konkuk University, Seoul \\ 05029, Republic of Korea \\ ${ }^{4}$ Research Institute of Medical Sciences, Konkuk University, Seoul 05029, Republic of Korea
}

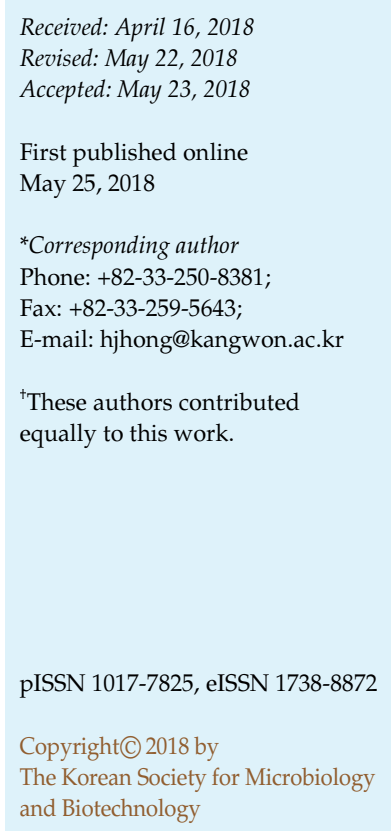

The hepatitis B virus (HBV) envelope contains small (S), middle (M), and large (L) proteins. PreS1 of the L protein contains a receptor-binding motif crucial for HBV infection. This motif is highly conserved among $10 \mathrm{HBV}$ genotypes (A-J), making it a potential target for the prevention of HBV infection. In this study, we successfully generated a neutralizing human monoclonal antibody $(\mathrm{mAb}), 1 \mathrm{~A} 8$ (IgG1), that recognizes the receptor-binding motif of preS1 using a phage-displayed human synthetic Fab library. Analysis of the antigen-binding activity of $1 \mathrm{~A} 8$ for different genotypes indicated that it can specifically bind to the preS1 of major HBV genotypes (A-D). Based on Bio-Layer interferometry, the affinity $\left(K_{\mathrm{D}}\right)$ of $1 \mathrm{~A} 8$ for the preS1 of genotype $C$ was $3.55 \mathrm{nM}$. 1A8 immunoprecipitated the hepatitis $B$ virions of genotypes $C$ and D. In an in vitro neutralization assay using HepG2 cells overexpressing the cellular receptor sodium taurocholate cotransporting polypeptide, 1A8 effectively neutralized HBV infection with genotype D. Taken together, the results suggest that $1 \mathrm{~A} 8$ may neutralize the four HBV genotypes. Considering that genotypes A-D are most prevalent, 1A8 may be a neutralizing human $\mathrm{mAb}$ with promising potential in the prevention and treatment of $\mathrm{HBV}$ infection.

Keywords: Hepatitis B virus, preS1, human monoclonal antibody, phage display, synthetic antibody library, neutralizing antibody

\section{Introduction}

Hepatitis B virus (HBV) infection is one of the most serious and prevalent public health problems. Approximately 257 million people are chronically infected with HBV, resulting in 887,000 deaths in 2015, mostly from complications of acute or chronic HBV infection, including liver cirrhosis and hepatocellular carcinoma [1]. To date, 10 distinct HBV genotypes (A-J) with different geographical distributions have been identified. Genotypes A and D are widespread in Africa and Europe; genotypes $B$ and $C$ are prevalent in
Asia; and genotypes E-J are occasionally encountered in Europe, America, and Asia. Genotypes A-D are most prevalent and are responsible for approximately $90 \%$ of hepatitis B cases globally [2].

The HBV envelope contains three structurally related small (S), middle (M), and large (L) proteins. The $S$ protein is the common C-terminal domain of these envelope proteins, the $\mathrm{M}$ protein consists of preS2 and $\mathrm{S}$, and the $\mathrm{L}$ protein consists of preS1, preS2, and S [3]. In addition to virions, HBV-infected hepatocytes also contain non-infectious spherical and filamentous subviral particles. These subviral 


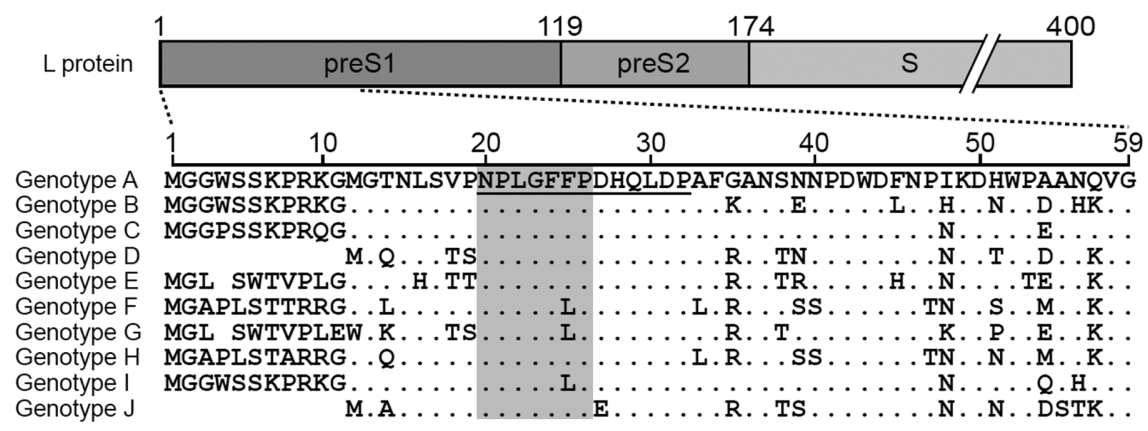

Fig. 1. Construction of recombinant preS1 antigens of different hepatitis B virus (HBV) genotypes.

Amino acid sequence alignment of the HBV preS1 N-terminal receptor-binding region (genotypes A-J, numbering based on HBV genotype A). The essential receptor-binding motif of preS1 (amino acids 20-26) is shaded in gray. The sequence contained in the Bio-preS1-L peptide is underlined.

particles consist mainly of $S$ proteins and typically outnumber virions by 1,000:1 to 10,000:1. Thus, these particles have been suspected to reduce the virus-specific immune response by mimicking virions [4]. Unlike $S$ protein, the preS1 of L protein exists primarily in infectious virions. In addition, amino acids 20-26 of preS1 play an essential role in the interaction with the cellular receptor sodium taurocholate cotransporting polypeptide (NTCP) and mediate HBV infection [5, 6]. This essential region is highly conserved among HBV genotypes (Fig. 1), making it a potential target for the prevention and treatment of $\mathrm{HBV}$ infection.

Hepatitis B immunoglobulin (HBIG) is currently used as a post-exposure prophylactic medical treatment for accidental or perinatal HBV exposure. HBIG is prepared by collecting the serum of high anti-S protein antibody titers. Considering that the preS1 region is crucial for $\mathrm{HBV}$ infection, a preS1-specific monoclonal antibody (mAb) may represent a promising approach for the prevention and treatment of HBV infection. Clinically, the appearance of anti-preS1 antibodies in patients correlates well with better recovery from acute hepatitis $B$ [7]. To date, several antipreS1 mAbs with HBV-neutralizing activity in vivo or in vitro have been generated by immunizing mice with $\mathrm{HBV}$ particles or recombinant preS1 antigen [8-14]. However, most of the antibodies do not neutralize all of the HBV genotypes. Therefore, we aimed to generate mAbs against the highly conserved essential region of preS1 for more effective prevention strategies. In the present study, using a phage-displayed human synthetic Fab library, we describe the successful generation of a human mAb, 1A8, which binds to the highly conserved receptor-binding motif of preS1 and neutralizes HBV infection in vitro.

\section{Materials and Methods}

\section{Cell Culture}

Suspension-adapted HEK293F cells (Invitrogen, USA) were grown in FreeStyle 293 Expression medium (Gibco, USA) at $125 \mathrm{rpm}$ and $37^{\circ} \mathrm{C}$ in a humidified incubator with $8 \% \mathrm{CO}_{2}$. Human hepatoma HepG2 cells were grown in DMEM (Gibco, USA) supplemented with $10 \%$ fetal bovine serum (GE Healthcare Life Sciences, USA) at $37^{\circ} \mathrm{C}$ in a humidified incubator with $5 \% \mathrm{CO}_{2}$.

\section{Preparation of PreS1 Antigens}

GST-preS1(1-119), GST-preS1(1-56), and preS1(1-119) of HBV genotype $C$ were prepared as described previously [15]. The recombinant preS1 of $\mathrm{HBV}$ genotypes $\mathrm{A}-\mathrm{G}$, comprising the Ig1-5 domains of human L1 cell adhesion molecule (L1CAM), preS1(160), and Strep tag, was transiently expressed in HEK293F cells for 7 days and purified by affinity chromatography using a Streptactin Superflow high capacity column (Iba, Germany) as described previously [9]. Biotinylated synthetic preS1 peptide (biotin-SGSGNPLGFFPDHQLDP, Bio-preS1-L peptide) containing the NTCP-binding motif (20-NPLGFFP-26) with $>98 \%$ of purity was purchased from AnyGen, Inc. (South Korea). The recombinant preS1 antigens and Bio-preS1-L peptide were used as antigens for biopanning and enzyme-linked immunosorbent assay (ELISA).

\section{Selection of PreS1-Specific Fab from a Phage-Displayed Human Synthetic Fab Library}

A human synthetic Fab library $\left(1.35 \times 10^{9}\right.$ diversity, unpublished $)$ constructed in our laboratory was panned against the four preS1 antigens GST-preS1(1-119), GST-preS1(1-56), Bio-preS1-L peptide, and preS1(1-119), for the first, second, third, and fourth rounds, respectively, using the standard panning procedure [16]. Briefly, an immunotube (or streptavidin-coated plates in the case of BiopreS1-L peptide) was coated with each preS1 antigen at $4^{\circ} \mathrm{C}$ overnight. Antibody library phages were incubated with the antigen and unbound phages were washed out with $0.1 \%$ PBST 
A

\begin{tabular}{ccccc}
\hline Round & Antigen & Wash & $\begin{array}{c}\text { Input phage } \\
\text { titer (pfu/ml) }\end{array}$ & $\begin{array}{c}\text { Output phage } \\
\text { titer (pfu/ml) }\end{array}$ \\
\hline $1 \mathrm{R}$ & GST-preS1 (1-119) & 10 & $1.62 \times 10^{14}$ & $1.42 \times 10^{6}$ \\
$2 \mathrm{R}$ & GST-preS1 (1-56) & 13 & $1.76 \times 10^{14}$ & $1.0 \times 10^{6}$ \\
$3 \mathrm{R}$ & PreS1 peptide & 15 & $3.10 \times 10^{13}$ & $6.33 \times 10^{7}$ \\
$4 \mathrm{R}$ & PreS1 (1-119) & 20 & $6.90 \times 10^{13}$ & $5.49 \times 10^{9}$ \\
\hline
\end{tabular}

B

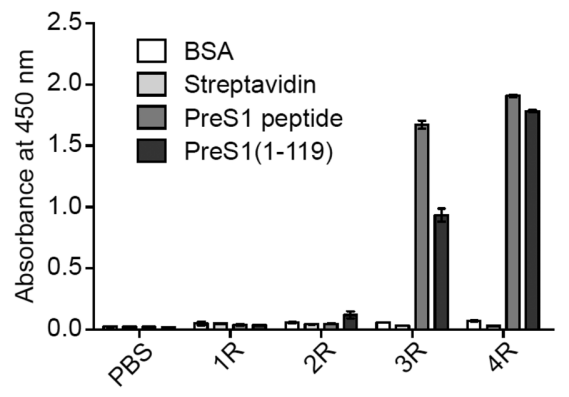

C

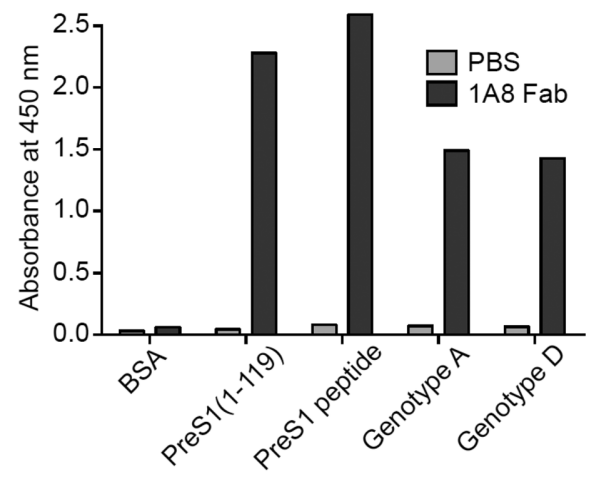

Fig. 2. Isolation of anti-preS1 monoclonal Fab from a human synthetic Fab library.

(A) Input and output phage titers of each panning round. pfu, plaque-forming unit. (B) Enrichment of positive polyclonal phage clones as determined by indirect ELISA. The fourth round of panning with maximal enrichment against preS1 was chosen for isolation of the monoclonal Fab. Values were obtained from duplicate wells and are expressed as the mean \pm SEM. (C) The antigen-binding activity of the 1A8 phage Fab was assessed by indirect ELISA using the preS1(1-119) of genotype C, Bio-preS1-L peptide, or recombinant preS1 of HBV genotypes A or D.

(0.1\% Tween 20 in PBS). The bound phages were eluted using $10 \mu \mathrm{g} / \mathrm{ml}$ trypsin solution at $37^{\circ} \mathrm{C}$ for $30 \mathrm{~min}$. Escherichia coli TG1 cells $\left(\mathrm{OD}_{600} \sim 0.5\right)$ were infected with the eluted phages, grown for $1 \mathrm{~h}$ at $37^{\circ} \mathrm{C}$, plated on a $2 \times \mathrm{YT} /$ carbenicillin/glucose agar plate, and incubated overnight at $37^{\circ} \mathrm{C}$. The amplified phages were subjected to the next round of panning, with the washing stringency increased gradually at each round (Fig. 2A). For titration, the number of input and output phages for each panning round was calculated by dilutions and colony-forming units. After the four rounds of panning, the output cells were grown and infected with helper phages to obtain polyclonal Fab phages. These phages were subjected to indirect ELISA to confirm the enrichment of positive clones. After the fourth round of panning, a total of 188 colonies were randomly selected and individually grown as monoclonal Fab phages, and then subjected to indirect and quantitative ELISAs. For indirect ELISA, 100 ng of BSA, BiopreS1-L peptide, preS1(1-119), or recombinant preS1 was used as an immobilized antigen and horseradish peroxidase (HRP)conjugated anti-M13 (1:5,000 (v/v); GE Healthcare, USA) as a secondary antibody. For quantitative ELISA, anti-M13 antibody (100 ng/well, GE Healthcare, USA) and anti-human kappa-HRP $(1: 2,000(\mathrm{v} / \mathrm{v})$; Novex, USA) were used as the immobilized antigen and secondary antibody, respectively, as described previously [17].

\section{Conversion of Fab into IgG1 and Expression in HEK293F Cells}

To convert the selected Fab into IgG1 format, the heavy chain variable region $(\mathrm{VH})$ and kappa light chain variable region (VK) sequences were amplified by PCR and combined with the IgG heavy and light chain leader sequences, respectively, using recombinant PCR. The resulting $\mathrm{VH}$ and $\mathrm{VK}$ sequences were subcloned into the EcoRI-ApaI and HindIII-BsiWI sites of the pdCMV-dhfrC expression plasmid containing the human $C \gamma 1$ and $\mathrm{C} \kappa$ genes, respectively. The resulting expression plasmids were introduced into HEK293F cells using polyethyleneimine (linear $25 \mathrm{kDa}$; Polysciences, USA) at a ratio of 1:4 (60 $\mu \mathrm{g}: 240 \mu \mathrm{g})$ as described previously [18]. The transfected cells were cultured for 7 days and the culture supernatants filtered using a bottle top filter (0.22 $\mu \mathrm{m}$ PES; Sartorius, Germany) for antibody purification.

\section{Purification of Anti-PreS1 Human mAb}

The culture supernatants were subjected to affinity chromatography on Protein A-agarose beads (Amicogen, Korea) as described previously [9]. The antibody concentration was determined by a NanoDrop 2000 UV-Vis Spectrophotometer (Thermo Fisher Scientific, USA). The integrity and purity of the purified antibody were assessed by sodium dodecyl sulfate polyacrylamide gel electrophoresis (SDS-PAGE).

\section{Analysis of Antigen-Binding Activities of Purified 1A8 mAb}

To analyze the antigen-binding activities of the purified 1A8 $\mathrm{mAb}$ to the preS1 antigens of different HBV genotypes, the 
recombinant preS1 antigen (200 ng) of different genotypes was individually incubated with anti-L1CAM antibody A10-A3 (200 ng) coated on each well, and then the bound preS1 antigen was incubated with serially diluted $1 \mathrm{~A} 8$ antibody. The bound $1 \mathrm{~A} 8$ antibody was detected using anti-human IgG Fc-HRP (1:10,000 $(\mathrm{v} / \mathrm{v})$; Jackson ImmunoResearch Laboratiries, USA) as the secondary antibody. To compare the antigen-binding activity between 1A8 and HzKR127-3.2, the antibody was serially diluted and incubated with recombinant preS1 (genotype C, $100 \mathrm{ng}$ ) coated on each well, and then the bound antibody was detected using anti-human IgG Fc-HRP (1:8,000 (v/v); Invitrogen, USA) as the secondary antibody.

\section{Affinity Determination}

For affinity determination of the antibody by Bio-Layer interferometry (BLI) using Octet RED (ForteBio, USA), antihuman Fc-coated biosensor tips (AHC, ForteBio, USA) were activated in $0.1 \%$ PBA ( $0.1 \%$ BSA in PBS) for $20 \mathrm{~min}$ by agitating in a 96-well microtiter plate (Greiner Bio-One, Austria) at 1,000 rpm. Antibody $(1 \mu \mathrm{g} / \mathrm{ml}, 200 \mu \mathrm{l})$ was captured for $10 \mathrm{~min}$ and washed with $0.1 \%$ PBA for $3 \mathrm{~min}$. Purified recombinant preS1 of $\mathrm{HBV}$ genotype A was prepared as a 2-fold serial dilution (100, 50, 25, 12.5 , and $6.25 \mathrm{nM}$ ) with $0.1 \%$ PBA and separately incubated with antibody bound on the tips. Association and dissociation rates were measured for 10 and $20 \mathrm{~min}$, respectively. All measurements were corrected for baseline drift by subtracting a control sensor (antibody-captured AHC sensor) exposed to running buffer only. The operating temperature was maintained at $30^{\circ} \mathrm{C}$. Data were analyzed using a 1:1 interaction model (fitting global, $R_{\max }$ unlinked by sensor) in ForteBio data analysis software ver. 7.1.

\section{Western Blot Analysis}

GST-preS1(1-56) was expressed in E. coli DH5a with $0.2 \mathrm{mM}$ isopropyl- $\beta$-D-thiogalactopyranoside (IPTG) for $2 \mathrm{~h}$, as described previously [19]. Cell lysates from uninduced or IPTG-induced cells and $10 \mu \mathrm{g}$ of purified GST-preS1(1-56) were separated by $12 \%$ SDS-PAGE, transferred to a nitrocellulose membrane, and incubated with antibody $(0.5 \mu \mathrm{g} / \mathrm{ml}, 10 \mathrm{ml})$, followed by antihuman IgG Fc-HRP conjugate (1:6,000 (v/v); Thermo Scientific, USA). The bands were visualized using a chemiluminescent substrate (WEST-ZOL plus, iNtRON BioTechnology, Korea).

\section{Immunoprecipitation Assay}

To produce HBV particles, HepG2 cells were seeded at a density of $6 \times 10^{5}$ cells in 6 -well plates and cultured at $37^{\circ} \mathrm{C}$. The next day, the HepG2 cells were transfected with pHBV5.2 (HBV genotype C) or pHBV1.2 (HBV genotype D) using Lipofectamine 2000 (Invitrogen, USA) [20, 21], and then incubated for 4 days. The culture supernatants were harvested and incubated with $1 \mu \mathrm{g} / \mathrm{ml}$ antibody overnight at $4^{\circ} \mathrm{C}$, and then immunoprecipitated with $20 \mu \mathrm{l}$ of protein A beads for $6 \mathrm{~h}$ at $4^{\circ} \mathrm{C}$. After washing the immunoprecipitated complex three times with PBS, the viral DNA was detected as described previously [20]. Briefly, the immunocomplex was treated with DNase I (Sigma, USA) and mung bean nuclease (Takara, Japan) at $37^{\circ} \mathrm{C}$ for $20 \mathrm{~min}$ to remove transfected plasmid DNA. The core-associated HBV DNA was prepared by digestion with Proteinase K $(20 \mathrm{mg} / \mathrm{ml}$; Roche, USA) at $37^{\circ} \mathrm{C}$ for $2-3 \mathrm{~h}$ in the presence of $0.5 \%$ SDS. HBV DNA was extracted using phenol/chloroform/isoamyl alcohol (25:24:1) (Sigma, USA) and precipitated with ethanol and $3 \mathrm{M}$ sodium acetate. The purified DNA was separated on a $1 \%$ agarose gel, and HBV DNA was detected by Southern blot hybridization using a ${ }^{32}$ P-labeled HBV probe [20, 22].

\section{In Vitro HBV Neutralization Assay}

The HBV-neutralizing activity of $1 \mathrm{~A} 8$ was investigated using a HepG2-NTCP stable cell line. The HepG2-NTCP cells were seeded at a density of $6 \times 10^{5}$ cells in 6-well plates and cultured at $37^{\circ} \mathrm{C}$. The next day, the HepG2-NTCP cells were infected with HBV particles of genotype D ( 2,000 viral genome equivalents per cell) in primary hepatocyte maintenance medium (PMM) containing $4 \%$ PEG and $2.5 \%$ DMSO as described previously [20]. For the neutralization assay, the HBV particles were pre-incubated with each antibody at different concentrations for $1 \mathrm{~h}$ at room temperature and then added to the cultured HepG2-NTCP cells. The medium was changed every 2 days for PMM supplemented with $2.5 \%$ DMSO, and the infected cells were harvested 7 days post-infection. The intracellular HBV DNA was extracted from the infected HepG2-NTCP cells and subjected to Southern blot hybridization.

\section{Results}

\section{Isolation of Fabs Specific to HBV PreS1 from a Phage Antibody Library}

To isolate Fabs specific to preS1 of HBV, a phagedisplayed human synthetic Fab library $\left(1.35 \times 10^{9}\right.$ diversity, unpublished) was panned against four preS1 antigens: GST-preS1(1-119), GST-preS1(1-56), Bio-preS1-L peptide, and preS1(1-119) [15]. The output phage titers increased after the third round of panning against the Bio-preS1-L peptide (Fig. 2A), and the antigen-binding activity of polyclonal output phages to preS1 antigens increased significantly, as assessed by indirect ELISA (Fig. 2B). After the fourth round of panning, 188 phage Fab clones were randomly selected and their antigen-binding activities analyzed by indirect ELISA using preS1(1-119), Bio-preS1-L peptide, and recombinant preS1 of genotypes $\mathrm{A}$ and $\mathrm{D}$. The 1A8 phage Fab had the highest antigen-binding activity with preS1 and was selected for further study (Fig. 2C).

\section{Conversion of 1A8 Fab into Human IgG1 and Analysis of Its Antigen-Binding Activity}

The 1A8 Fab was converted into human IgG1 and 
A
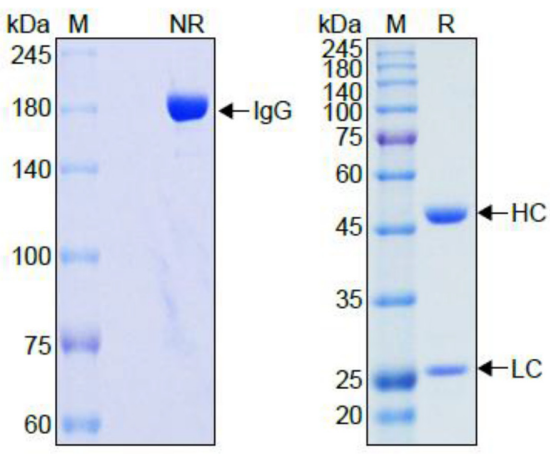

C

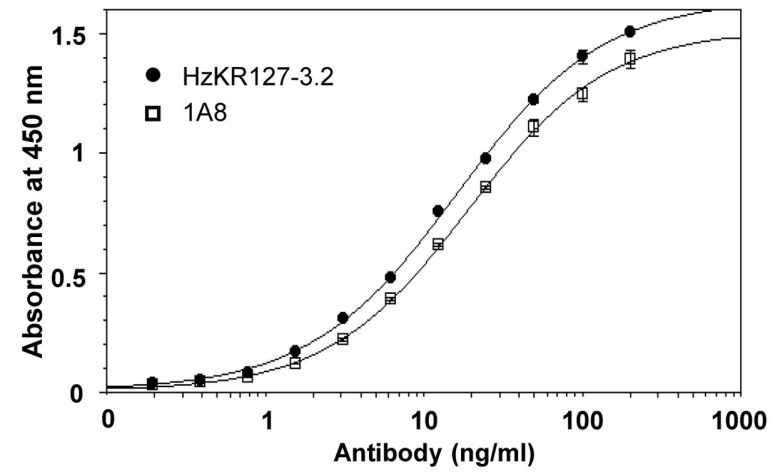

B

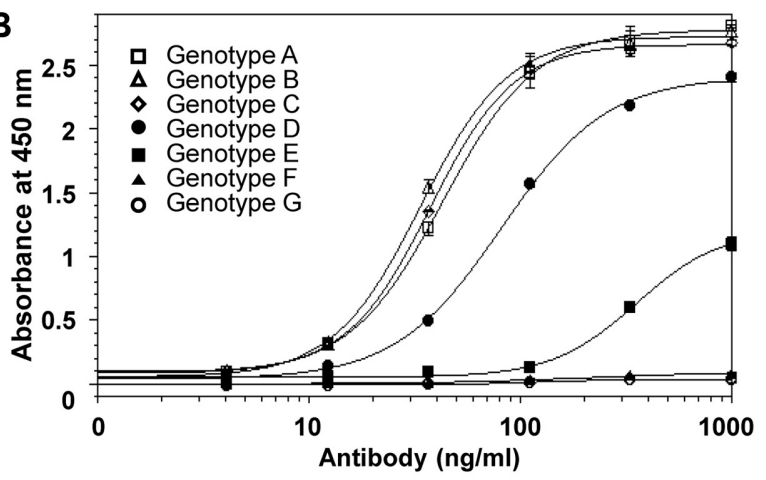

D

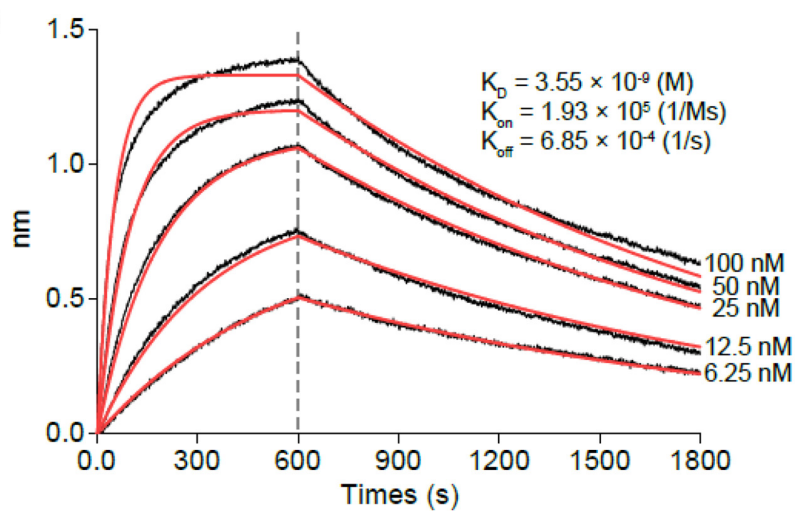

Fig. 3. Analysis of the antigen-binding activity of the purified $1 \mathrm{~A} 8 \mathrm{mAb}$.

(A) SDS-PAGE of purified 1A8 under non-reducing (NR, 6\%) and reducing $(\mathrm{R}, 10 \%)$ conditions. M, protein marker; HC, heavy chain; LC, light chain. (B) The antigen-binding activities of purified $1 \mathrm{~A} 8 \mathrm{mAb}$ to the preS1 of different HBV genotypes (A-G) were assessed by ELISA. (C) The antigen-binding activity of purified 1A8 was compared with that of humanized anti-preS1 mAb HzKR127-3.2 by indirect ELISA using the recombinant preS1 of HBV genotype C. (D) Affinity determination of 1A8 by Bio-Layer interferometry using Octet RED. The recombinant preS1 of HBV genotype A was prepared as a 2-fold serial dilution (100, 50, 25, 12.5, and $6.25 \mathrm{nM})$. All values were obtained from duplicate wells and are expressed as the mean \pm SEM.

transiently expressed in HEK293F cells, and the culture supernatants were subjected to protein purification. The integrity and purity of purified 1A8 IgG1 were confirmed by SDS-PAGE (Fig. 3A). The purified $1 \mathrm{~A} 8$ bound to the recombinant preS1 of genotypes $\mathrm{A}-\mathrm{D}$; its antigen-binding activity to genotypes $\mathrm{A}-\mathrm{C}$ was the same and approximately $35 \%$ higher than to genotype D (Fig. 3B). However, 1A8 hardly bound to preS1 of genotype $\mathrm{E}$, and did not bind to genotypes $\mathrm{F}$ and $\mathrm{G}$. In addition, the antigen-binding activity of $1 \mathrm{~A} 8$ to preS1(1-119) of genotype C was slightly lower than that of HzKR127-3.2 [8], a humanized antipreS1 $\mathrm{mAb}$ with a $K_{\mathrm{D}}$ of $0.5 \mathrm{nM}$ (Fig. 3C). Affinity determination by BLI using Octet RED indicated that the $K_{\mathrm{D}}$ of $1 \mathrm{~A} 8$ for the recombinant preS1 of genotype A was approximately $3.55 \mathrm{nM}$ (Fig. 3D).

\section{Validation of the Antigen-Binding Specificity of 1A8}

To evaluate the antigen-binding specificity of $1 \mathrm{~A} 8$, GST-
preS1(1-56) was expressed in E. coli DH5a and the lysates of uninduced or induced cells were subjected to western blot analysis for 1A8, with purified GST-preS1(1-56) as a positive control. As shown in Fig. 4, 1A8 exhibited antigenbinding specificity to preS1 without any nonspecific binding activity to endogenous protein of E. coli.

\section{Evaluation of the HBV-Neutralizing Activity of 1A8}

Prior to assessing the in vitro HBV-neutralizing activity of $1 \mathrm{~A} 8$, an immunoprecipitation assay was performed to determine whether $1 \mathrm{~A} 8$ can bind the hepatitis $\mathrm{B}$ virion. A previously developed HBV-neutralizing humanized antipreS1 mAb, HzKR127-3.2, was used as a positive control for HBV genotypes $C$ and D, whereas the humanized antipreS1 mAb HzKR359-1 was used as a positive control for genotype $C$ and a negative control for genotype $D[8,9]$. Mouse IgG was used as a negative control. Viral DNA was extracted from immunoprecipitates and measured by 


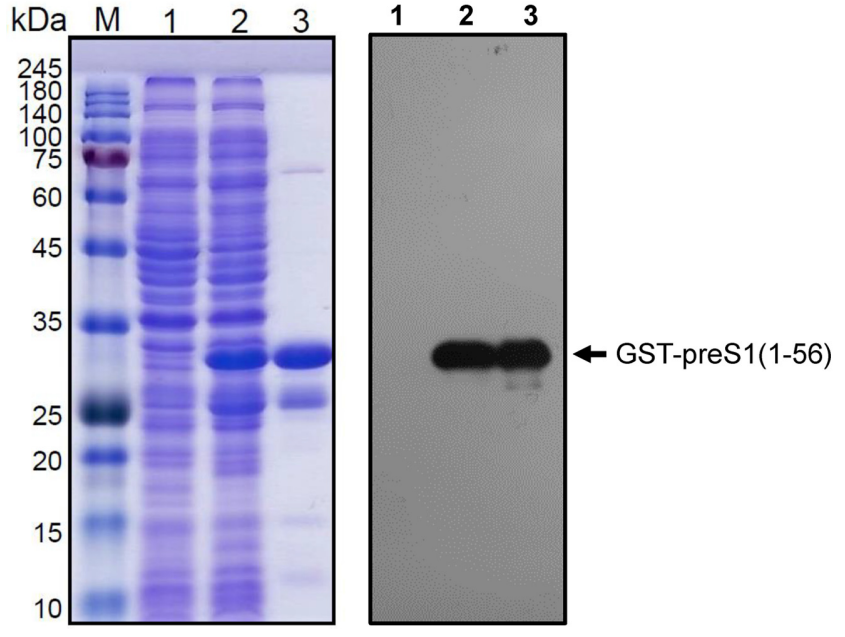

Fig. 4. Western blot analysis of $1 \mathrm{~A} 8 \mathrm{mAb}$.

GST-preS1(1-56) was expressed in E. coli DH5a and subjected to $12 \%$ SDS-PAGE (left) followed by western blot analysis with 1A8 (right). $\mathrm{M}$, protein marker; lane 1, uninduced cell lysates; lane 2, IPTGinduced cell lysates; lane 3, purified GST-preS1(1-56) with the molecular mass of $34 \mathrm{kDa}$.

Southern blot hybridization. As shown in Fig. 5A, the relaxed circular (RC) and double-stranded linear (DSL) forms of HBV DNA were detected in 1A8 precipitates, indicating that $1 \mathrm{~A} 8$ could bind to both HBV genotypes $\mathrm{C}$ and D.

To examine whether $1 \mathrm{~A} 8$ can neutralize HBV infection, HBV particles of genotype D were pre-incubated with each antibody at different concentrations, and then added to cultured HepG2-NTCP cells overexpressing the cellular receptor NTCP. At 7 days post-infection, the infected cells were harvested and the intracellular HBV DNA was measured by Southern blot hybridization. Although 1A8 had a lower binding activity to preS1 of genotype D than to that of genotype C (Fig. 3B), it effectively neutralized infection with both HBV genotypes in a dose-dependent manner (Fig. 5B). The neutralizing efficacy was similar to that of HzKR127-3.2, which has a higher affinity than 1A8. This result is consistent with the immunoprecipitation assay.

\section{Discussion}

PreS1 of HBV contains a highly conserved receptorbinding motif that plays an essential role in and mediates HBV infection. However, HBIG, which is currently used as a post-exposure prophylactic medical treatment, contains mostly anti-S protein antibodies. The subviral particles are usually present in 10,000-fold excess over complete HBV particles in the blood of infected persons. Therefore, mAbs targeting the receptor-binding motif of preS1 may represent a promising approach for the effective prevention and treatment of HBV infection. In this study, we successfully generated a broadly neutralizing human $\mathrm{mAb}$ specific to the receptor-binding motif by panning a phage-displayed human synthetic Fab library against preS1 antigens comprising the short peptide containing the receptorbinding motif. Analysis of the antigen-binding activity indicated that $1 \mathrm{~A} 8$ can bind to the preS1 of four major HBV genotypes (A-D). In addition, 1A8 bound the HBV particles of both genotypes $\mathrm{C}$ and $\mathrm{D}$ and effectively neutralized infection with $\mathrm{HBV}$ of genotype $\mathrm{D}$ in vitro. The results suggest that $1 \mathrm{~A} 8$ may neutralize HBV genotypes A-D. Given that these genotypes are responsible for approximately
A

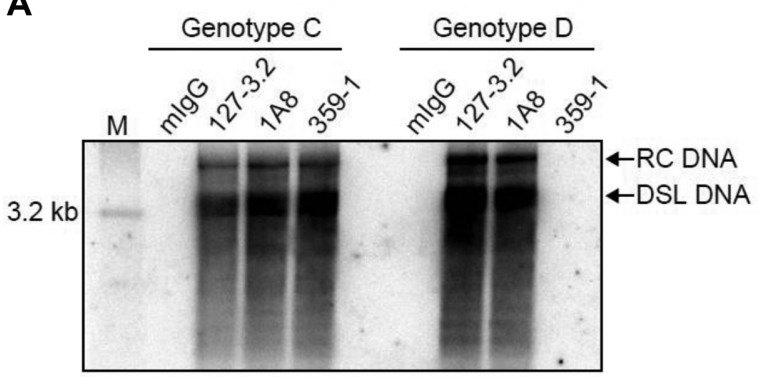

B

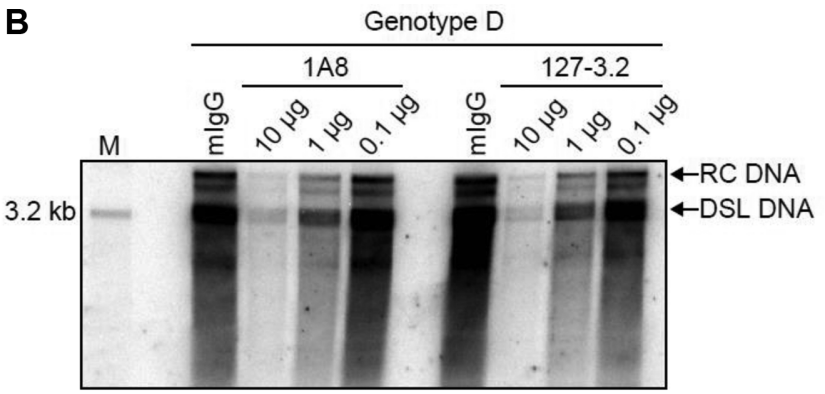

Fig. 5. Evaluation of the virion-binding activity and in vitro virus-neutralizing activity of $1 \mathrm{~A} 8$ against $\mathrm{HBV}$ of genotype $\mathrm{D}$.

(A) Immunoprecipitation of HBV particles (genotypes C and D) by 1A8. HzKR127-3.2 was used as a positive control for genotypes C and D, whereas HzKR359-1 was used as a positive control for genotype $C$ and a negative control for genotype D. Mouse IgG was used as a negative control for both genotypes. RC DNA, relaxed circular DNA; DSL DNA, double-stranded linear DNA. (B) HBV particles (genotype D) were preincubated with 1A8 or HzKR127-3.2 (10, 1, and $0.1 \mu \mathrm{g})$, and then added to cultured HepG2-NTCP cells. The medium was changed every 2 days and infected cells were harvested at 7 days post-infection. The intracellular HBV DNA was extracted and subjected to Southern blot hybridization. 
$90 \%$ of hepatitis B cases globally [2], 1A8 may represent a neutralizing human $\mathrm{mAb}$ that can be used for the prevention and treatment of HBV infection.

Regarding the epitope of $1 \mathrm{~A} 8$, it exhibited reduced binding activity towards preS1 of genotypes $\mathrm{D}$ or $\mathrm{E}$ compared with genotypes $\mathrm{A}-\mathrm{C}$, indicating that the amino acid at position 19 influences the antigen-binding activity of $1 \mathrm{~A} 8$, and that Pro19 is optimal for antigen binding. In addition, 1A8 did not exhibit antigen-binding activity toward preS1 of genotypes F or G, indicating that Phe 25 is essential for antibody binding. The results suggest that $1 \mathrm{~A} 8$ recognizes amino acids $20-25$ in the receptor-binding motif and, thus, neutralizes HBV infection by blocking the preS1NTCP interaction.

To date, several murine anti-preS1 mAbs with HBVneutralizing activity in vivo or in vitro have been reported, but these antibodies exhibit limited neutralizing activities against some HBV genotypes, in addition to the immunogenicity issue [12-14]. In our previous study, we developed HBV-neutralizing humanized anti-preS1 mAb HzKR127-3.2 and HzKR359-1 [8, 9]. HzKR127-3.2 exhibited binding activity only to preS1 of genotypes $A, C, D$, and $G$, whereas HzKR359-1 bound to genotypes $A, B$, and $C$. To our best knowledge, $1 \mathrm{~A} 8$ is the first human anti-preS1 $\mathrm{mAb}$ that may neutralize the four major HBV genotypes (A-D). Considering that the presence of anti-preS1 antibodies in patients correlates with better recovery from acute hepatitis B, and that antibody-mediated immunotherapy could be effective for chronic hepatitis B [7, 23], 1A8 may be effective in the prevention of HBV infection and as a therapy for hepatitis B.

\section{Acknowledgments}

This work was supported by a grant (C1011479-01-03) from the Ministry of Science, ICT and Future Planning.

\section{Conflict of Interest}

The authors have no financial conflicts of interest to declare.

\section{References}

1. WHO. 2017. Global Hepatitis Report 2017. World Health Organization, Geneva, Switzerland.

2. Sunbul M. 2014. Hepatitis B virus genotypes: global distribution and clinical importance. World J. Gastroenterol. 20: 5427.

3. Ganem D, Prince AM. 2004. Hepatitis B virus infection - natural history and clinical consequences. N. Engl. J. Med. 350: 1118-1129.

4. Huang C-F, Lin S-S, Ho Y-C, Chen F-L, Yang C-C. 2006. The immune response induced by hepatitis $\mathrm{B}$ virus principal antigens. Cell. Mol. Immunol. 3: 97-106.

5. Schulze A, Schieck A, Ni Y, Mier W, Urban S. 2010. Fine mapping of pre-S sequence requirements for hepatitis $B$ virus large envelope protein-mediated receptor interaction. J. Virol. 84: 1989-2000.

6. Yan H, Zhong G, Xu G, He W, Jing Z, Gao Z, et al. 2012. Sodium taurocholate cotransporting polypeptide is a functional receptor for human hepatitis B and D virus. Elife 1: e00049.

7. Wei J, Wang Y-Q, Lu Z-M, Li G-D, Wang Y, Zhang Z-C. 2002. Detection of anti-preS1 antibodies for recovery of hepatitis B patients by immunoassay. World J. Gastroenterol. 8: 276.

8. Kim JH, Gripon P, Bouezzedine F, Jeong MS, Chi S-W, Ryu S-E, et al. 2015. Enhanced humanization and affinity maturation of neutralizing anti-hepatitis B virus preS1 antibody based on antigen-antibody complex structure. FEBS Lett. 589: 193-200.

9. Wi J, Jeong MS, Hong HJ. 2017. Construction and characterization of an anti-hepatitis B virus preS1 humanized antibody that binds to the essential receptor binding site. $J$. Microbiol. Biotechnol. 27: 1336-1344.

10. Maeng C-Y, Ryu CJ, Gripon P, Guguen-Guillouzo C, Hong HJ. 2000. Fine mapping of virus-neutralizing epitopes on hepatitis B virus preS1. Virology 270: 9-16.

11. Hong HJ, Ryu CJ, Hur H, Kim S, Oh HK, Oh MS, et al. 2004. In vivo neutralization of hepatitis $B$ virus infection by an anti-preS1 humanized antibody in chimpanzees. Virology 318: 134-141.

12. Heermann K, Goldmann U, Schwartz W, Seyffarth T, Baumgarten H, Gerlich W. 1984. Large surface proteins of hepatitis B virus containing the pre-S sequence. J. Virol. 52: 396-402.

13. Pizarro JC, Vulliez-le Normand B, Riottot M-M, Budkowska A, Bentley GA. 2001. Structural and functional characterization of a monoclonal antibody specific for the preS1 region of hepatitis B virus. FEBS Lett. 509: 463-468.

14. Zhang P, Yu WMY, Venable R, Alter HJ, Shih JW-K. 2006. Neutralization epitope responsible for the hepatitis $\mathrm{B}$ virus subtype-specific protection in chimpanzees. Proc. Natl. Acad. Sci. USA 103: 9214-9219.

15. Kim HS, Hong HJ. 1995. Efficient expression, purification and characterization of hepatitis B virus preS1 protein from Escherichia coli. Biotechnol. Lett. 17: 871-876.

16. Kontermann R, Dübel S. 2010. Antibody Engineering. Vol. 2. Springer, Berlin/Heidelberg.

17. Yoon J-Y, Kim D-H, Kim S, Kim D, Jo G, Shin M-S, et al. 2017. Generation of a monoclonal antibody that has reduced binding activity to VX-inactivated butyrylcholinesterase (BuChE) compared to BuChE by phage display. Biotechnol. 
Bioprocess Eng. 22: 114-119.

18. Backliwal G, Hildinger M, Hasija V, Wurm FM. 2008. Highdensity transfection with HEK-293 cells allows doubling of transient titers and removes need for a priori DNA complex formation with PEI. Biotechnol. Bioeng. 99: 721-727.

19. Maeng C-Y, Oh MS, Park IH, Hong HJ. 2001. Purification and structural analysis of the hepatitis B virus preS1 expressed from Escherichia coli. Biochem. Biophys. Res. Commun. 282: 787-792.

20. Park YK, Park E-S, Kim DH, Ahn SH, Park SH, Lee AR, et al. 2016. Cleaved c-FLIP mediates the antiviral effect of TNF- $\alpha$ against hepatitis $\mathrm{B}$ virus by dysregulating hepatocyte nuclear factors. J. Hepatol. 64: 268-277.

21. Ryu CJ, Gripon P, Park HR, Park SS, Kim YK, GuguenGuillouzo C, et al. 1997. In vitro neutralization of hepatitis B virus by monoclonal antibodies against the viral surface antigen. J. Med. Virol. 52: 226-233.

22. Southern EM. 1975. Detection of specific sequences among DNA fragments separated by gel electrophoresis. J. Mol. Biol. 98: 503-517.

23. Zhang T-Y, Yuan Q, Zhao J-H, Zhang Y-L, Yuan L-Z, Lan Y, et al. 2015. Prolonged suppression of HBV in mice by a novel antibody that targets a unique epitope on hepatitis $B$ surface antigen. Gut 65: 658-671. 